\title{
DEVELOPMENT AND VALIDATION OF THE PSYCHOSOCIAL ISSUES OF HYSTERECTOMY CANCER PATIENT SCALE
}

\author{
Tanzila Tanzeem \\ PhD scholar \\ University of Management and Technology \\ tanzilat@skm.org.pk

\section{Zahid Mahmood} \\ Adjunct Professor, School of Professional Psychology, Department of Clinical Psychology, \\ University of Management and Technology \\ zahid.mahmood@umt.edu.pk
}

\begin{abstract}
This research was aimed to develop a measure of Psychosocial Issues of Hysterectomy Cancer Patient Scale. For this purpose, psychosocial issues were explored from 160 ovarian cancer patients using mix method research design (both quantitative and qualitative) was used to explore the mental health functioning emotional, psychological and psycho-social concerns, behavioral changes, material relationship within the context of Pakistani society. A list of 27 items was converted into a self-report measure psychosocial issue of hysterectomy. Factor analysis was applied to collected data of 160 participants. KMO was found to be 0.87 whereas Bartlett's test of sphericity was significant at .000 level and found out to have acceptable psychometric properties.
\end{abstract}

KEYWORDS: Psychosocial Issues, Cancer, Scale Development, Hysterectomy

\section{INTRODUCTION}

Pakistan is a third world country and there is a need to investigate the different potential elements of troubles that accompanied malignancy. In man centric culture of Pakistan a few issues of females go unaddressed, especially with ovarian disease. Talking about marital life, ladies experience sporadic relational encounters. A female needs to absolutely depend on her close accomplice, for being living in man centric framework. Ovarian disease can open a female to shaky conjugal life, dread of being surrender by spouse and second marriage by him. Things become more troublesome if the female accomplice has no kids before finding (particularly ovarian malignancy) that clear way for conjugal disagreement and sometimes separation. The examination endeavors to disclose these covered issues to aid satisfactory strategy development, determination of suitable treatment convention, utilization of fundamental psychosocial mediations to guarantee psychological well-being working and a native assortment of information to enlighten their experience of the sickness (Faisal, Muhammad, Rafshan, Sajid, Sajida \& Shahid, 2019).

Malignancy is developing now daily and hitting numerous ladies from one side of the planet to the other. Having a malignancy itself is exceptionally startling and makes ladies more unfortunate with regards to their lives. The therapy for ovarian malignancy is additionally an exceptionally difficult technique. It absolutely modifies the self-perception of ladies; on the grounds that during and after treatment the progressions happen to like going bald, weight gain and misfortune, menopause indications, and more significant and greater misfortune is the evacuation of a body organ. Ovaries are the image of gentility and sexuality since civilization started. Expulsion of the uterus makes ladies more cognizant with regards to their self-perception. Patients with ovarian malignant growth who went through hysterectomy medical procedure experienced self-perception unsettling influence (Chen, Liao, Chen, Chan \& Chen, 2020). 
According to (World Health Organization, 2021) defines "Cancer is the uncontrolled development and spreads of cells. The developments often attack nearby tissue and can metastasize to distant sites". Hysterectomy, the removal of the uterus, is the leading reason for non-obstetric surgery among women in many high-income settings (Stankiewicz, 2019). It can be done by abdominal or vaginal route and with help of laparoscopy. Hysterectomy is the effective treatment option for many conditions like fibroid, abnormal uterine bleeding, endometriosis, uterine prolapse, and pelvic inflammatory disease and in some cases of genital tract malignancies (Saravana, Patil \& Patil, 2019).

\section{REVIEW OF LITERATURE}

A number of causative factors have been reported for development of ovarian cancer, however some established and more vulnerable factors include family history and age of patients (Al-Badawi, Munkarah, Tulbah, Babic, \& Husaini, 2018). Also those women who have difficulty in becoming pregnant, or experience a normal menopause with hot flashes have high risk of developing the disease (Kota, Gayatri, Pani, Meher \& Modi, 2021). Intake of fats is another risk factor of developing ovarian cancer (Sadeghi, Shab-Bidar, Parohan \& Djafarian, 2019). In Pakistan, very limited cancer data have been published that is quite insufficient to present any Incidence or prevalence rates. However, a survey was conducted for the period 1995-1997 from the population of the Karachi South district comprising than, a population of 1.7 million. Overall, $95.3 \%$ of the incident cases were microscopically verified. The reported incidence rates for all cancers combined were 80.5 per 100,000 for males and 91.8 per 100,000 for females (Bhurgri, Hassan, Zaidi, Rahim \& Sankaranarayanan, 2020).

Individuals at the time of diagnosis, serious pain, absence of social support, propelling infection, additionally are at high risk to create nervousness issue during treatment (Price, Butow, Costa, King, Aldridge, Fardell, DeFazio \& Webb, 2019). The advancement of depression among individuals with ovarian cancer is additionally prone to rely upon factors at the structural level, including insurance expenses and access to welfare support as cancer can have a huge financial impact (Gilligan, Alberts, Roe \& Skrepnek, 2018; Lu, Sullivan \& Sharp, 2019). Undeniably, females and reproduction go side by side that's the reason womb is symbol of identity (Leppert, Legro \& Kjerulff, 2019). The females are overwhelmed by fear because they believe it will lead to rejection and their sexual life will be destroyed (Dragisic \& Milad, 2018; Sung, \& Lim, 2020). Cancer is considered to be a deadly and chronic disease in this era which leads towards the short and long term difficulties related to psychological problems, physical disabilities and sexual problems (Patterson, Moylan, Bannon \& Salih, 2020)

Keskin and Gumus (2017) there are many subsets of psychological symptoms referred to in the literature. These are: anxiety and depression attributed to the operation, sexual dysfunction (presenting as diminished libido, pain, dyspareunia or anxiety surrounding sexual activity) and reactions related to perceptions of feminist and low self-esteem. Turkish females experiencing hysterectomy encountered a few sexual issues, for example, veganism's, low recurrence and no interest of sex and poor association with their companion. In a meta-investigation improvement in scores of uneasiness, gloom in females experiencing hysterectomy (Darwish, Atlantis, \& Mohamed, 2017). Examinations led a detailed an expansion in post-hysterectomy uneasiness, tension and burdensome indications in Nigerian and Egypt females (Okunlola, Umuerri, Omigbodun, Morhason-Bello, Okonkwo, \& Ojengbede, 2018).

Hysterectomy is much of the time performed gynecological treatment in Pakistan. This surgery is an exceptionally unpleasant occasion for Pakistani females experiencing extreme gynecological ailments and intricacies. It is likewise connected with a high rate of tension and gloom. A few examinations in the West have announced an improvement in the side effects of sadness and uneasiness in patients experiencing hysterectomy. Yet, this circumstance may be distinctive in under developed nations like Pakistan where it is normally connected with, hazardous inconveniences and obstetric variations from the norm. Mental help for these females is nearly non-existent by and large careful settings (Raza, Waqas, \& Jamal, 2017). Entanglements to an exceptional socio-cultural setting of Pakistan, insufficient maternity, absence of appropriate referral framework, unbooked status, blundered work, ignorance of the patient, family and Dais (untrained birthing assistants) (Shaikh et al., 2020). In her examination, Farooqi (2017) detailed a critical increment in scores on depression and anxiety in patients at week post-hysterectomy. 
Though, Shah et al., (2017) detailed a high frequency of uneasiness (69.6\%) and discouragement (14.4\%) however a diminishing pattern in anxiety and depression scale scores at 24 weeks' post-hysterectomy.

In spite of the fact that the hysterectomy seems to have a low mortality with one death per thousand, there is critical morbidity related with the method with a quarter to half of all female experiencing the system building up some dreariness, fever and drain being the most widely recognized (Akhtar, Asif \& Roshan, 2017). Late continuation may incorporate the lingering ovary disorder, depression and an expanded danger of cardiovascular illness (Aziz, 2019). Mental issues may happen following hysterectomy, the most every now and again revealed being anxiety and depression (Ahsan, \& Naeem, 2021). A few investigations have indicated a noteworthy relationship between hysterectomies and depression (Vandyk, Brenner, Tranmer, \& Van Den Kerkhoff, 2021). This mental comorbidity is related to the impression of low self-perception and loss of parenthood (Flory, Bissonnette, \& Binik, 2019). A total of 100 females took an interest in research recurrence of uneasiness and melancholy in members was as per the following: Anxious (62\%), fringe on edge (29\%), discouraged (36\%) and fringe discouraged $(46 \%)$. None of them were accepting any sort of mental help. Age of the respondent, number of kids and higher uneasiness scores were fundamentally connected with scores on discouragement subscale (Raza, Waqas, \& Jamal, 2017).

\section{RESEARCH METHODOLOGY}

The research was carried out into 4 phase that aimed to explore the essence and meaning of the lived experiences of post hysterectomy suffering of Pakistani women as well as devising a standardized measure of Psychosocial Issues of Hysterectomy scale.

\section{Phase I: Item Generation}

Through purposive sampling total 40 participant for ovarian cancer were recruited who underwent hysterectomy. The age range of the sample is 25 to 60 years. Their consent was taken and 60 minutes were allotted to each individual. Along with this on field notes were also recorded. The data saturation was done at 35 participants, interviews of 5 more participants were taken for the counter verification process. Inductive approach of content analysis was used to extract significant statements, eliminate irreverent repetition, discarding statements not revelatory of the phenomenon and identify the central themes or meaning implicit in statements. In the start 90 common statements identified regarding hysterectomy. After excluding the repetition and ambiguity 50 items were finalized with 4-point rating scale.

\section{Phase II Opinion by the subject experts and Patient Opinion}

Through convenient sampling 5 experienced clinical psychologists were selected with minimum 5 years' experience and working in psycho-oncology field asked them to rate each item to the extent to which it reflects on a 4-point rating scale. After expert evaluation in order to remove ambiguity, changes were done and a final list of 28 items were converted into a self-report measure for hysterectomy patients. This Performa was given to 10 other hysterectomy patients. They were individually asked if this list catered to all their concerns to which they gave their approval.

\section{Phase III Pilot study (Try out)}

Agreed self-report inventory for hysterectomy was given to the 35 participants for piloting to assess the user friendliness at the actual pollution on the same inclusion \& exclusion criteria. The purpose of this phase is to remove those items that are rejected by participants or those face validity and content validly does not match. After conducting pilot study 12 items were replacing due to complex wording or language to reduce down ambiguity.

\section{Phase IV: Main Study}

The homogeneous sample of 160 female hysterectomy patients were selected through purposive sampling from Shaukat Khanum Memorial Cancer Hospital and Research Center (SKMCH \&RC) and Anmol cancer hospital. Sample was recruited in two ways: out patients and in patient receiving medical active treatment. The participants were informed about the purpose of the study. The participants of the study were with the age range of 25 to $60(\mathrm{M}=43.01, \mathrm{SD}=7.61)$. Women with definite diagnosis of 
ovarian cancer who underwent hysterectomy who had 3 months passed to surgery with active treatment phase were included in the sample.

\section{MEASURING TOOLS \\ Demographic Performa}

This Performa included the basic characteristic information based on age, education, timespan of treatment, married/unmarried and hysterectomy cause is cancerous/non-cancerous.

\section{Psychosocial Issues of Hysterectomy}

Indigenously developed Psychosocial Issues of Hysterectomy scale for ovarian cancer that underwent with hysterectomy was consisted of 28 items designed to measure psychosocial issues. Can be rated on 4point Likert scale range from 0 to 3 where 0 is "never", 1 is "very less", 2 is "Somewhat/some extent" and 3 is "mostly/ very much". The instructions for the scale were "Following are some statements that are given by subjects like you. Read them carefully how much do they apply to you".

\section{Depression Anxiety Stress Scale (DASS).}

The DASS was used which consisted of 21 items designed to measure the negative emotional states of depression, anxiety and stress. Can be rated on 4-point Likert scale range from 0 to 3 where 0 is "never", 1 is "very less", 2 is "Somewhat/some extent" and 3 is "mostly/ very much" (Lovibond, \& Lovibond, 1995).

\section{PROCEDURE}

Non-probability purposive sampling technique was used. The researcher assured the participant about the confidentiality of all the information. The majority of the participants showed their consents to fill the questioners. The participants were given a demographic information sheet, psychosocial issues of hysterectomy scale and DASS. Total 160 ovarian cancer participants were included in the research. The questionnaire were distributed to the control group and experimental groups of hysterectomy patients to check out the effectiveness of the therapies and activities on psychosocial issues of hysterectomy, provided during the six sessions for 45 minutes over a period of six weeks. After data collection questionnaires were scored according to the scoring procedures. The quantitative analysis and interpretation of the results were reported.

\section{RESULTS}

Factor Analysis of Psychosocial Issues of Hysterectomy Scale Assessing the Data and Extracting the Factors.

After pilot study the finalized items of the psychosocial issues of hysterectomy the underlying factor structure was determined by obtaining a principal component solution. The exploratory factor analysis was applied to the data obtained from 160 participants during the main study. The researcher has used Kaiser Criterion to find the components that have an Eigenvalue of more than 1 (Kaiser, 1974). KaiserMeyer-Olkin (KMO) was found to be 0.87 and Bartlett's test of sphericity was found out to be significant at .000 level.

By using the Kaiser criterion too many components are extracted so it is important to look at the scree plot provided by the output. According to the assumption there is a need to check the curvy linear relationship, change or elbow in the shape of the scree plot; components above this point are retained (Kaiser, 1974). Component 1 explains the much more variance than the remaining components. There was a quite small break between the 2 and 3 components from this plot it is recommended to extract only 2 components. The final table need to consider is the component matrix it shows the loading of each item on the 2 components. We can see from this table that all the items except last two items load quite strongly above .4. It supports our conclusion from scree plot to retain only 2 components for further investigation. 


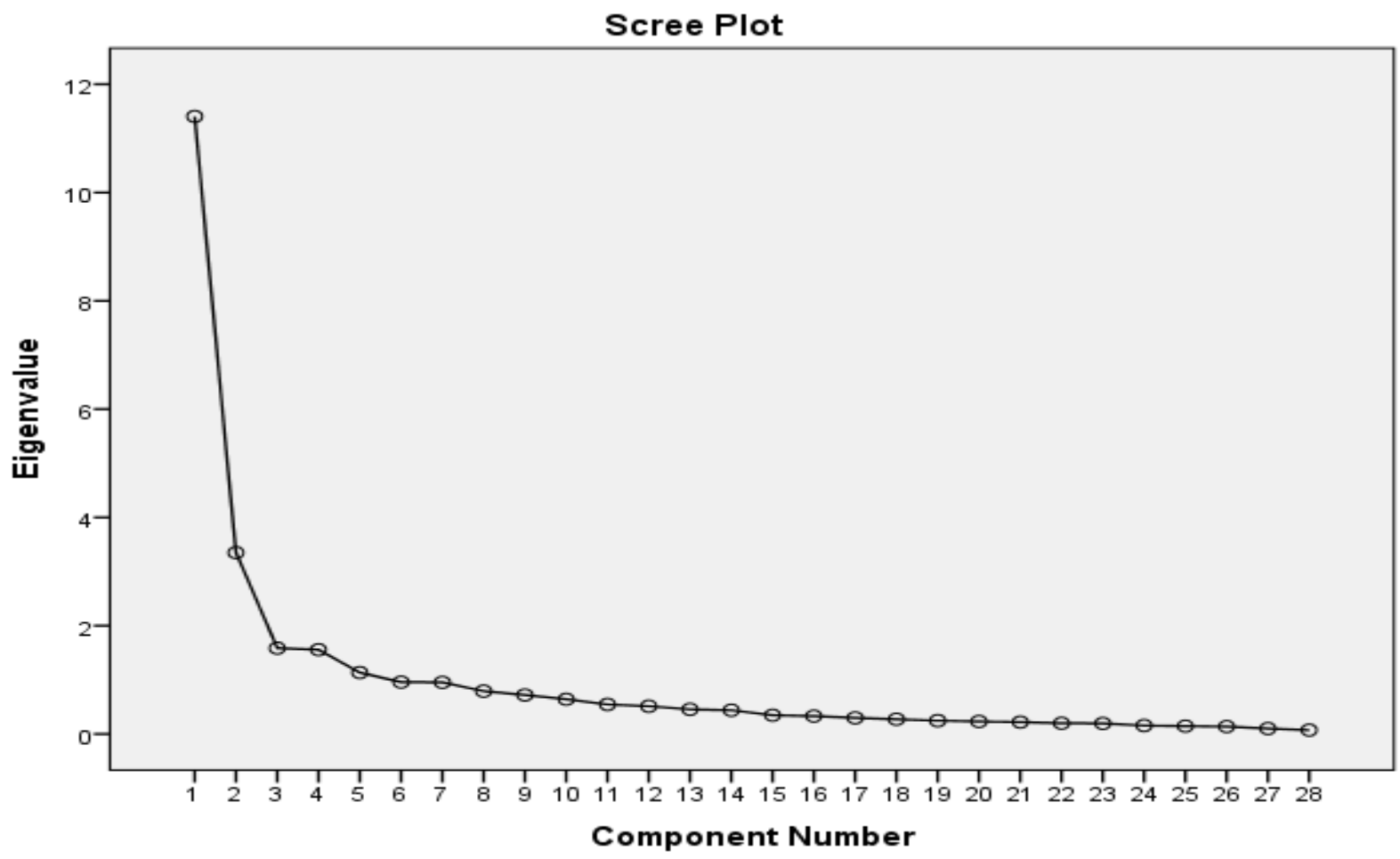

Figure 1. Scree Plot of Factor Loadings of Psychosocial Issues of Hysterectomy Scale

Factor Rotation and Interpretation. Once the number of factors has been determined, the next step is to interpret them. For this process the factors are rotated. Only 2 components extracted and rotated using varimax rotation technique. In the rotated component matrix the highest loading of each of the variables on the two factors was observed, which helped in identifying the nature of latent variable represented by each component.

Factor 1(Interpersonal). Interpersonal factor consisted of 14 items like "people attitude towards me get change" "people's sympathies make me feel pity" "After hysterectomy I feel like my respect in people's view ended" and etc.

Factor 2 (Depression). This is the second factor of the scale consisted of 13 items like "feeling of sadness" "feeling of irritability or fatigue" "tension" "negative thinking" "weakness in body" and etc.

\section{Table 1}

Factor Structure and Eigen Values of Psychosocial Scale of Hysterectomy with Varimax Rotation $(N=160)$.

\begin{tabular}{|c|c|c|c|}
\hline Sr No. & Item No. & Factor 1 & Factor 2 \\
\hline 1. & $16 \mathrm{PSH}$ & .82 & \\
\hline 2. & 14PSH & .82 & \\
\hline 3. & 15PSH & .80 & \\
\hline 4. & $19 \mathrm{PSH}$ & .80 & \\
\hline 5. & 21PSH & .77 & \\
\hline 6. & $23 \mathrm{PSH}$ & .76 & \\
\hline 7. & $20 \mathrm{PSH}$ & .75 & \\
\hline 8. & $22 \mathrm{PSH}$ & \multirow{4}{*}{\multicolumn{2}{|c|}{$\begin{array}{l}.72 \\
.70 \\
.66\end{array}$}} \\
\hline 9. & $13 \mathrm{PSH}$ & & \\
\hline 10. & $25 \mathrm{PSH}$ & & \\
\hline 11. & $18 \mathrm{PSH}$ & & \\
\hline 12. & $28 \mathrm{PSH}$ & \multicolumn{2}{|c|}{.64} \\
\hline 13. & $27 \mathrm{PSH}$ & \multicolumn{2}{|c|}{.61} \\
\hline
\end{tabular}


14.

15.

16.

17.

18.

19.

20.

21.

22.

23.

24.

25.

26.

27.

28.

Eigen Values

$\%$ of Variance

$\%$ of Cumulative
$17 \mathrm{PSH}$

$11 \mathrm{PSH}$

$3 \mathrm{PSH}$

$1 \mathrm{PSH}$

$2 \mathrm{PSH}$

$5 \mathrm{PSH}$

$4 \mathrm{PSH}$

$7 \mathrm{PSH}$

$9 \mathrm{PSH}$

$8 \mathrm{PSH}$

$10 \mathrm{PSH}$

$24 \mathrm{PSH}$

$12 \mathrm{PSH}$

$6 \mathrm{PSH}$

26PSH
.62

.85
.83
.79
.76
.71
.64
.63
.57
.56
.46
.45
.43
.41
3.35
40.74
52.69

.85

83

.76

.71

64

.63

.57

.56

.45

.43

.41

11.41

40.74

40.74

11.96 52.69

Note. Items with .4 or above are bold faced

Table 1, showing the final two factors solution of the scale, factors were extracted with the loading of .4 and above. For the purpose of factor extraction, factor analysis with 2, 3 and 4 factor solution was tried. Hence the suitable factor analysis with minimum dubious values was kept, which was with 2 factors. The item number 11 was cross load on two factor with no value so it was dropped from the original scale so the total remaining items come out to be 27 . Thus, the factor 1 of the scale contains 14 items and factor 2 contains 13 items of total 27 items of the scale.

\section{Psychometric Properties of Psychosocial Issues of Hysterectomy Scale}

Table 2

Psychometric properties of Psychosocial Issues of Hysterectomy Scale $(N=160)$.

\begin{tabular}{llll}
\hline Factor & $\mathrm{K}$ & $\mathrm{M}(\mathrm{S} . \mathrm{D})$ & Alpha $(a)$ \\
\hline 1.Interpersonal & 14 & $24.75(11.29)$ & .94 \\
2.Depression & 13 & $24.54(8.13)$ & .88 \\
3.TPSH & 27 & $51.11(17.95)$ & .94 \\
\hline
\end{tabular}

Note. $\mathrm{K}=$ no. of items, $\mathrm{M}=$ Mean, $\mathrm{S} . \mathrm{D}=$ Standard deviation, $a=$ Cronbach's alpha, TPSH=Total of psychosocial scale of hysterectomy

The overall alpha reliability of the psychosocial issues of Hysterectomy scale was .94. Its subscale interpersonal has reliability value .94 and second subscale has reliability value of .88 .

Table 3

Inter-correlations, Means, and Standard Deviation for the Subscales of Psychosocial Issues of Hysterectomy Scale $(N=160)$.

\begin{tabular}{llll}
\hline Factors & 1.Interpersonal & 2.Depression & TPSH \\
\hline $1 . \mathrm{IP}$ & - & $.91^{* *}$ & $.84^{* *}$ \\
2.Dep & & & $.56^{* *}$ \\
3.TPSH & & - & - \\
$M$ & 24.75 & 24.54 & 51.11
\end{tabular}


Note. $\mathrm{M}=$ Mean, $\mathrm{S} . \mathrm{D}=$ Standard deviation, $a=$ Cronbach's alpha, TPSH=Total of psychosocial scale of hysterectomy. $* * \mathrm{p}<.01$

Table 3, describes the Pearson product moment correlation in order to determine the strength of relation between the factors of PSH and the total of PSH. The results indicated that all the factors in psychosocial scale of hysterectomy have significant positive correlation. Further, the results also showed that the total of PSH also has positive correlation with its factors such as interpersonal $(\mathrm{r}=.84, p<.01)$ and depression $(\mathrm{r}=.56, p<.01)$.

\section{Concurrent Validity Analysis}

Concurrent validity was established by exploring the correlation between newly developed Psychosocial issues of Hysterectomy scale with already developed well standardized DASS.

\section{Table 4}

Correlation of Total Hysterectomy Scale with Depression, Anxiety, Stress Scale (N=160).

\begin{tabular}{lllll}
\hline Variables & Hysterectomy & Interpersonal & Depression & DASS \\
\hline Hysterectomy & & $.82^{* *}$ & $.38^{*}$ & $.56^{* *}$ \\
Interpersonal & - & .21 & .23 \\
Depression & & - & $.57^{* *}$ \\
DASS & & & -
\end{tabular}

Note $=* \mathrm{p}<.05, * * \mathrm{p}<.01$

The results of table 4, indicates that DASS (Depression, anxiety, stress scale) correlates positively and significantly with newly developed Hysterectomy scale $(r=.56, P<.01)$ and also with its subscale (depression) $(r=.57, P<.01)$. The subscale of interpersonal has shown non-significant relationship with DASS.

\section{DISCUSSION}

The findings of this research are discussed with the support of previous literature related to this research. The purpose of the research was to assess psychosocial issues faced by the women's who experienced the hysterectomy for this reason the researcher had to develop an indigenous scale to measure the psychosocial issues of the hysterectomy cancer patient. This is the first attempt to develop a scale to measure the psychosocial issues of the hysterectomy cancer patients in Pakistani cultural context. The items of the scale were generated empirically through content analysis method (Shan, \& Yan, 2015).

It is clear from previous researches that women face several psychosocial issues like depression as a result of hysterectomy. A research was conducted that showed that hysterectomy had serious drawbacks on patients' self-confidence and consciousness. These are hardships and restrictions in physical and psychological scenarios experienced by patients after hysterectomy. The health care provider must be understanding and considerate about all these things in order to provide better health services (Alshawish, Qadous \& Yamani, 2020). Furthermore, one another research concluded that women after hysterectomy have feelings of mutilated body that adversely affect identity and functionality. It was postulated that instead of biological care the patent should be provided with multidimensional care (Silva \& Vargens, 2017).

The exploratory factor analysis of psychosocial issues of hysterectomy scale revealed 2 factor solution. Varimax rotation method is the simplest method to use, which helps in the prediction of the precise and clear interpretation of each factor (Kaiser, 1974). The two factors of indigenously developed psychosocial issues of hysterectomy scale (Interpersonal, Depression) were quite similar to the already developed well standardized Depression Anxiety stress scale (DASS). The psychosocial issues of hysterectomy was compared with DASS and reliability of psychosocial issues of hysterectomy scale was .94. Its subscale interpersonal has reliability value .94 and second subscale has reliability value of .88 


\section{CONCLUSION}

Hysterectomy is considered to pose different severe psychological, social and behavioral issues in women especially in Pakistan where the females are suppressed to talk about such issues. This research was done to explore psychosocial issues that most Pakistani women faced due to hysterectomy and offers to construct a measure for first time in the context of Pakistan. As it is constructed in Pakistani setting it will be culturally friendly to assess issues. The identification of this scale will specify the issues particularly related to the cancer context that will help in developing solutions to counter such problems. Stakeholders of Pakistan, policy maker and administrative can arrange workshops and provide training programs to cater this will give support to future researches in the field of sport (Williams, \& Clark, 2020).

\section{LIMITATIONS AND SUGGESTION.}

The limitation of the research was limited sample size due to the limited availability of samples. The sample was taken only from Lahore Shukat khanam hospital and Anmol cancer hospital so the findings cannot be generalized. The data should be collected from different cities of Pakistan to get large sample and diverse data for the validation of the scale. This topic is not well researched in Pakistan a longitudinal research on the experiences of hysterectomy cancer patients should be conducted to find out predictors of psychological, physical and psychosocial issues of hysterectomy cancer patients (Yoo, Levine, Aviv \& Ewing, 2020).

\section{IMPLICATIONS}

This is the examination in the area of oncology and its extreme impact on women issues research in Pakistan. The improvement of psychosocial issues of hysterectomy scale explicitly for the hysterectomy disease patients was a commitment in the field of mental appraisal. The substance investigation helps in definite examination of open finished reactions and gave the chance to sum up them completely and gives us a hull of psychosocial issues looked by the Pakistani ladies' determined to have hysterectomy. The Health and mental divisions of Pakistan can utilize the consequences of this investigation to deal with the psychological well-being of the patients by furnishing them with different intellectual rebuilding and stress the executive's procedures. Additionally, these discoveries lay justification for future investigates. With an all the more all-around planned procedure.

\section{REFERENCES}

Ahsan, S., Naeem, S., \& Ahsan, A. (2001). A case notes analysis of hysterectomy performed for nonneoplastic indications at Liaquat National Hospital, Karachi. Journal-Pakistan Medical Association, 51(10), 346-348.

Akhtar, S., Asif, S., \& Aoshan, R. (1996). Adenomyosis: a study of 3030 cases. J Coll Physicians Surg Pak, 6, 260-1.

Al-Badawi, I. A., Munkarah, A. R., Tulbah, A., Babic, I. I., Al Husaini, H., \& Ahmad, S. (2013). A detailed study of patients and tumor characteristics of epithelial ovarian cancer in Saudi women. International Journal of Gynecologic Cancer, 23(3).Alshawish, E., Qadous, M.S., \& Yamani, M.A. (2020). Experience of Palestinian Women After Hysterectomy Using a Descriptive Phenomenological Study. The Open Nursing Journal, 14(1).

Bhurgri, Y., Bhurgri, A., Hassan, S. H., Zaidi, S. H. M., Rahim, A., Sankaranarayanan, R., \& Parkin, D. M. (2000). Cancer incidence in Karachi, Pakistan: first results from Karachi cancer registry. International journal of cancer, 85(3), 325-329.

Chen, C. L., Liao, M. N., Chen, S. C., Chan, P. L., \& Chen, S. C. (2012). Body image and its predictors in breast cancer patients receiving surgery. Cancer nursing, 35(5), E10-E16.

Darwish, M., Atlantis, E., \& Mohamed-Taysir, T. (2014). Psychological outcomes after hysterectomy for benign conditions: a systematic review and meta-analysis. European Journal of Obstetrics \& Gynecology and Reproductive Biology, 174, 5-19. 
Dragisic, K. G., \& Milad, M. P. (2004). Sexual functioning and patient expectations of sexual functioning after hysterectomy. American journal of obstetrics and gynecology, 190(5), 1416-1418.Faisal, G., Muhammad, S. A., Rafshan, S., Sajid, B., Sajida, J., \& Shahid, M. B. (2019).

Gulzar, F., Akhtar, M. S., Sadiq, R., Bashir, S., Jamil, S., \& Baig, S. M. (2019). Identifying the reasons for delayed presentation of Pakistani breast cancer patients at a tertiary care hospital. Cancer management and research, 11, 1087.

Farooqi, Y.N. (2017). Depression and anxiety in patients undergoing hysterectomy. J Pakistan Psychiatric Society, 2, 13-6.

Flory, N., Bissonnette, F., \& Binik, Y. (2017). Psychosocial effects of hysterectomy -Literature review. Journal of psychosomatic research. 59. 117-29. 10.1016/j.jpsychores.2005.05.009

Gilligan, A. M., Alberts, D. S., Roe, D. J., \& Skrepnek, G. H. (2018). Death or debt? National estimates of financial toxicity in persons with newly-diagnosed cancer. The American journal of medicine, 131(10), 1187-1199.

Kaiser, H. F. (1974). An index of factorial simplicity. Psychometrika, 39(1), 31-36.

Keskin G., Gumus, A. B. (2017). Turkish hysterectomy and mastectomy patients -depression, body image, sexual problems and spouse relationships. Asian Pac J Cancer Prev, 12: 425-32.

Kota, S. K., Gayatri, K., Pani, J. P., Meher, L. K., Kota, S. K., \& Modi, K. D. (2012). Ovarian granulosa cell tumor: An uncommon presentation with primary amenorrhea and virilization in a pubertal girl. Indian journal of endocrinology and metabolism, 16(5), 836.

Leppert, P. C., Legro, R. S., \& Kjerulff, K. H. (2007). Hysterectomy and loss of fertility: implications for women's mental health. Journal of Psychosomatic research, 63(3), 269-274.

Lovibond, S.H. \& Lovibond, P.F. (1995). Manual for the Depression Anxiety \& Stress Scales. (2nd Ed.)Sydney: Psychology Foundation.

Lu, L., O'Sullivan, E., \& Sharp, L. (2019). Cancer-related financial hardship among head and neck cancer survivors: Risk factors and associations with health-related quality of life. Psycho-oncology, 28(4), 863-871.

Okunlola, M. A., Umuerri, C., Omigbodun, O. O., Morhason-Bello, I. O., Okonkwo, S. N., \& Ojengbede, O. A. (2018). Pattern of mental ill health morbidities following hysterectomy for benign gynecological disorders among Nigerian women. Int J Ment Health Syst. 3(1):18.

Patterson, P., Moylan, E., Bannon, S., \& Salih, F. (2020). Needs analysis of cancer education program in South Western Sydney. Cancer Nursing, 23, 186-92

Price, M.A., Butow, P.N., Costa, D.S., King, M.T., Aldridge, L.J., Fardel, J.E, \& DeFazio, A. (2019). Prevalence and predictors of anxiety and depression in women with invasive ovarian cancer and their caregivers. Med J Aust 193 (5):52-57

Raza, N., Waqas, A., \& Jamal, M. (2017). Post-operative anxiety, depression and psychiatric support in patients undergoing hysterectomy: A cross sectional survey. Jpma. the Journal of the Pakistan Medical Association, 65, 4, 443-5

Sadeghi, A., Shab-Bidar, S., Parohan, M., \& Djafarian, K. (2019). Dietary Fat Intake and Risk of Ovarian Cancer: A Systematic Review and Dose-Response Meta-Analysis of Observational Studies. Nutrition and cancer, 71(6), 939-953.

Saravana, A., Patil, S.B., Patil, S.S. (2019). Clinic pathological study of hysterectomised specimens. Int J Reprod Contracept Obstet Gynecol.6(1):246-8.

Shah, S.,Wagan, M.A., \& Saud, S.(2017). Incidence of anxiety and depression in women undergoing hysterectomy. Pak J Pharmacol; 24: 23-8.

Shaikh, N. B, Shaikh, S., \& Shaikh, J. M. (2020). Morbidity and mortality associated with obstetric hysterectomy. J Ayub Med Coll Abbottabad; 22: 100-4.

Shan-Shan H, \& Yan, L. (2015). Association between alcohol consumption and the risk of ovarian cancer: a metaanalysis of prospectiveobservational studies. BMC public health 15: 223

Silva, C. D. M. C., \& Vargens, O. M. D. C. (2017). A mulher que vivencia as cirurgias ginecológicas: enfrentando as mudanças impostas pelas cirurgias. Revista Latino-Americana de Enfermagem, 24. 
Stankiewicz A, Pogany L, Popadiuk C. (2019). Prevalence of self-reported hysterectomy among Canadian women. Chronic Diseases and Injuries in Canada 34: 30-5.

Sung, M.H, \& Lim, Y.M. (2020). Factors Affecting Sexual Satisfaction in Korean Women who have undergone a Hysterectomy .Australian Journal of Advanced Nursing, 27(2), 46-54.

Vandyk, A. D, Brenner, I., Tranmer, J., \& Van Den Kerkhoff, E. (2021). Depressive symptoms before and after elective hysterectomy. J Obs Gynecol Neonatal Nurs.1; 40: 566-76.

Williams, R.D., \& Clark, A.J. (2020). A qualitative study of Women's Hysterectomy Experience. Journal of Women's Health, 9(2): 15- 25.

World Health Organization. (2021). Cancer. Retrieved from http://www.who.int/topics/cancer/en/index.html

Yoo, G.J., Levine E.G., Aviv, C., Ewing C. \& Au. A. (2020). Older women, breast cancer, and social support. Support Care Cancer. 18, 1521-1530.Doi: 10.1007/s00520-009-0774-4 